\title{
Analysis of Vibration Characteristics for Overhead Transmission Line based on the Finite Model
}

\author{
Zhang Guojun* \\ INNER MONGOLIA EHV POWER SUPPLY BUREAU,CHINA \\ wslsd5030@163.com
}

Keywords: transmission lines; finite element model;Euler beam; modal; vibration characteristics

Abstract.Considering bending stiffness,an finite element model is proposed to overhead transmission based on Euler beam.In this model,the displacement of the unit beam is represented by assumed mode method,and derived the mass matrix and stiffness matrixof unit beam by using assumed mode method,secondly,deduced Lagrange equation using d'Alembert principle and vibration differential equation of unit beam.And then the concept of global coordinates is proposed,which is used to represent the entire transmission line system composed of several unit beam.Taking the LGJ-400/95 as the research object,the inherent characteristics of natural frequencies and mode shapes of the transmission lines and the vibration response under the loads of the different forms can be obtained by using the finite element model to analysis the vibration characteristics of the overhead transmission lines.

\section{Introduction}

Overhead transmission line has the characteristic of long span crossing,big flexible, low damping. Large displacement and small strain is normal in the working condition.It is senitive to the low frequency wind loads.Wind-induced vibration of transmission lines is a complex non-linear dynamic problems.

The paper establish a finite element model based on Euler beam for modal analysis and vibration response analysis of the transmission lines.The main feature of this model is to analysis the bending stiffness of the transmission lines,rather than simplify look the wire as flexible cable. Dividing the transmission line into a number of Euler beam,which is the basic idea to establish the model,and using the assumed mode to represent the displacement of each beam element nodes. derived the mass matrix and stiffness matrixof unit beam by using assumed mode method,secondly,deduced Lagrange equation using d'Alembert principle and vibration differential equation of unit beam. And then the concept of global coordinates is proposed, which is used to represent the entire transmission line system composed of several unit beam.

\section{The derivation of vibration equation of the unit beam}

The establishment of a unit beam vibration deflection curve equation Assuming Unit beam segment of length $L$ under in literal node force, which the deflection curve shown in Figure 1. 


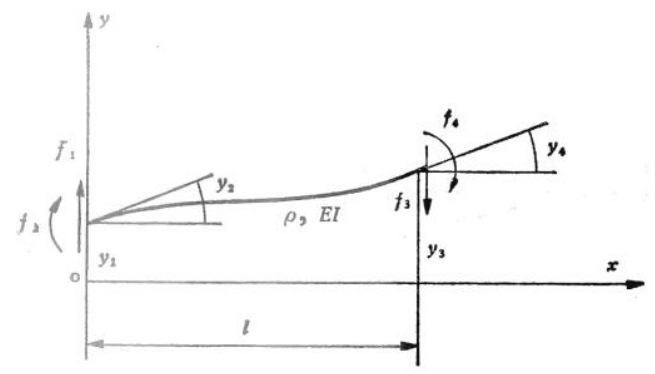

Figure 1. Unit beam deflection curve

Distributed load of beam set zero,the differential equation of the deflection of beam:

$$
\frac{d^{4} y}{d x^{4}}=0
$$

The form of the solution:

$$
y(x)=a_{0}+a_{1} x+a_{2} x^{2}+a_{3} x^{3}=X(x) \alpha
$$

In which, $X(x)=\left[1, x, x^{2}, x^{3}\right], \alpha=\left[a_{0}, a_{1}, a_{2}, a_{3}\right]$

The derivative of the formula (2):

$$
y^{\prime}(x)=a_{1}+2 a_{2} x+3 a_{3} x^{2}=X^{\prime}(x) \alpha
$$

Taking the deflection and corner of the beam element at the both ends as 4 generalied coordinates.

$$
y=\left\{\begin{array}{c}
y_{1} \\
y_{2} \\
y_{3} \\
y_{4}
\end{array}\right\}=\left\{\begin{array}{c}
y(0) \\
y^{\prime}(0) \\
y(l) \\
y^{\prime}(l)
\end{array}\right\}=C \alpha
$$

In which,

$$
\alpha=\left[\begin{array}{llll}
a_{0} & a_{1} & a_{2} & a_{3}
\end{array}\right], \quad C=\left[\begin{array}{cccc}
1 & 0 & 0 & 0 \\
0 & 1 & 0 & 0 \\
1 & l & l^{2} & l^{3} \\
0 & 1 & 2 l & 3 l^{3}
\end{array}\right]
$$

Which is obtained by the formula (4):

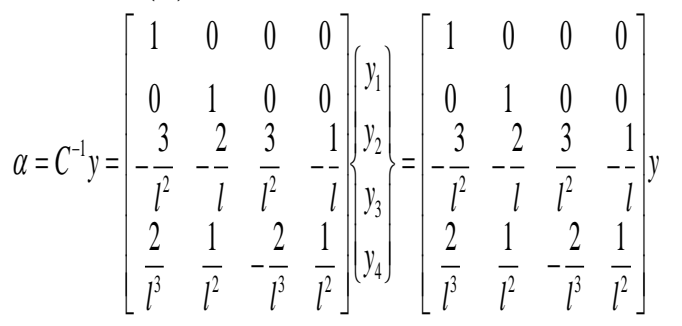

Substituting equation (5) into the formula (2) to obtain:

$$
y(x)=X(x) \alpha=X C^{-1} y=\phi(x) y
$$

In which,

$$
\begin{gathered}
\phi(x)=X C^{-1}=\left[\begin{array}{llll}
\phi_{1} & \phi_{2} & \phi_{3} & \phi_{4}
\end{array}\right] \\
\phi_{1}=1-3\left(\frac{x}{l}\right)^{2}+2\left(\frac{x}{l}\right)^{3} \\
\phi_{2} / l=\frac{x}{l}\left(1-\frac{x}{l}\right)^{2} \\
\phi_{3}=3\left(\frac{x}{l}\right)^{2}-2\left(\frac{x}{l}\right)^{3}
\end{gathered}
$$




$$
\phi_{4} / l=\left(\frac{x}{l}\right)^{2}\left(\frac{x}{l}-1\right)
$$

In the beam bending vibration, if it is still taking $\mathrm{y}$ as the generalized coordinates, $\mathrm{y}$ will be a function of time,the deflection lines will not only be a function of the postion $\mathrm{x}$, but also a function of $\mathrm{t}$. The deflection curve equation of beamVibrations:

$$
y(x, t)=X(x) \alpha(t)=\phi(x) y(t)
$$

\section{The calculation of unit beam quality matrix}

The lateral vibration speed at $\mathrm{t}$ of the beam at $\mathrm{x}$ is $\dot{y}_{i}=(x, t)$, as shown in Figure 2 :

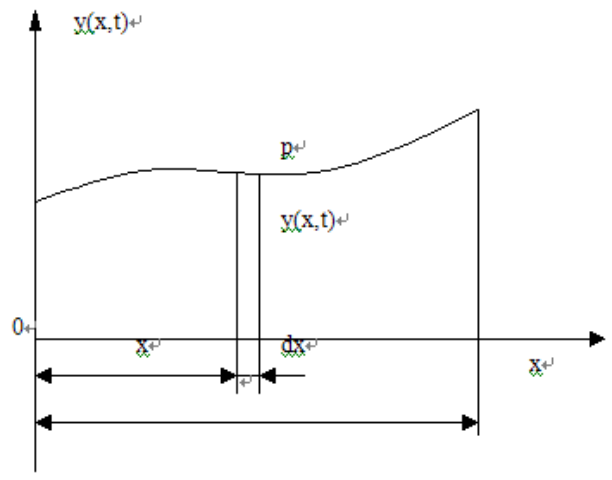

Figure 2. The kinetic energy of the micro beam segment

The kinetic energy of the micro beam segment:

$$
d T=\frac{1}{2} \dot{y}_{i}(x, t) d m=\frac{1}{2} \dot{y}_{i}(x, t) \rho d x
$$

The total kinetic energy of the beam:

$$
T=\frac{1}{2} \int_{0}^{l}\left[\rho \dot{y}_{i}^{2}(x, t) d x\right]
$$

The unit beam mass matrix is derived from unit beam kinetic energy:

$$
T=\frac{1}{2} \int_{0}^{l} \rho\left(\frac{\partial y}{\partial t}\right)^{2} d x=\frac{1}{2} \dot{y}^{T} \int_{0}^{l} \rho \phi^{T} \phi d x \dot{y}=\frac{1}{2} \dot{y}^{T} m \dot{y}
$$

Where $\mathrm{m}$ is the mass matrix of unit beam:

$$
m=\int_{0}^{l} \rho \phi^{T} \phi d x=\frac{\rho l}{420}\left[\begin{array}{cccc}
156 & 22 l & 54 & -13 l \\
22 l & 4 l^{2} & 13 l & -3 l^{2} \\
54 & 13 l & 156 & -22 l \\
-13 l & -3 l^{2} & -22 l & 4 l^{2}
\end{array}\right]
$$

The calculation of unit beam stiffness matrix

Bending deformation energy of mirco beam segment, as shown in Figure 3:

$$
d U=\frac{M^{2}(x)}{2 E I} d x
$$




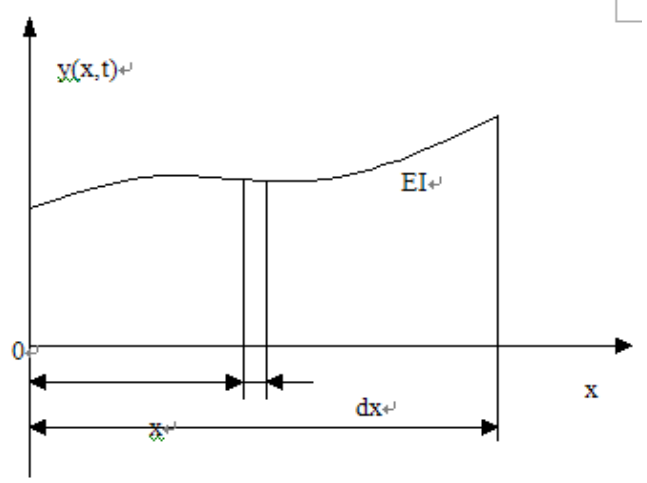

Figure 3. Bending deformation energy of the mirco beam segment The total bending deformation energy of the beam:

$$
U=\int_{0}^{l} \frac{M^{2}(x)}{2 E I} d x
$$

The unit beam stiffness matrix is derived from unit beam bending deformation energy:

$$
U=\frac{1}{2} \int_{0}^{l} E I\left(\frac{\partial^{2} y}{\partial x^{2}}\right) d x=\frac{1}{2} y^{T} k y
$$

Where $k$ is the stiffness matrix of unit beam:

$$
k=\int_{0}^{l} E I \phi^{\top T} \phi^{\prime \prime}=\frac{E I}{l^{3}}\left[\begin{array}{cccc}
12 & 6 l & -12 & 6 l \\
6 l & 4 l^{2} & -6 l & 2 l^{2} \\
-12 & 6 l & 12 & -6 l \\
6 l & 2 l^{2} & -6 l & 4 l^{2}
\end{array}\right]
$$

\section{The establishment of unit beam vibration differential equations}

Lagrange equation of the non-conservation system:

$$
\frac{d}{d t}\left(\frac{\partial L}{\partial \dot{q}_{i}}\right)-\frac{\partial L}{\partial q_{i}}=Q_{i}
$$

Where $\mathrm{L}$ called the momentum of the system is the difference of kinetic energy $\mathrm{T}$ and the potential energy U.i.e. $\mathrm{L}=\mathrm{T}-\mathrm{U}$.

$$
\begin{aligned}
& \frac{d}{d t}\left[\frac{\partial(T-U)}{\partial \dot{y}}\right]-\frac{\partial(T-U)}{\partial y}=Q \\
& \Rightarrow \frac{d}{d t}\left[\frac{\partial}{\partial \dot{y}}\left(\frac{1}{2} \dot{y}^{T} m \dot{y}\right)-\frac{\partial}{\partial \dot{y}}\left(\frac{1}{2} y^{T} k y\right)\right] \\
&-\frac{\partial}{\partial y}\left(\frac{1}{2} \dot{y}^{T} m \dot{y}\right)+\frac{\partial}{\Im}\left(\frac{1}{2} y^{T} k y\right)=Q
\end{aligned}
$$

In which,

$$
\frac{\partial U}{\partial \dot{y}}=\frac{\partial}{\partial \dot{y}}\left(\frac{1}{2} y^{T} k y\right)=0 \frac{\partial T}{\partial y}=\frac{\partial}{\partial y}\left(\frac{1}{2} \dot{y}^{T} m \dot{y}\right)=0
$$

Thus launched:

$$
m \ddot{y}+k y=Q
$$




\section{The derivation of transmission line model vibration equation}

Looking the transmission line as a system composed of number of unit beam. We introduce the concept of local coordinates and global coordinates, the four generalized coordinates from unit beam perspective called local coorinate, which the number of local coordinates is $4 \mathrm{n}$, and generalized coordinates defined from the whole system is called global coordinates, which the number of global coordinates is $2 \mathrm{n}+2$.

The corresponding generalized coordinates in the local coordinate is $y_{1 i}, y_{2 i} \ldots \ldots y_{n i}(i=1,2,3,4)$; we define the mass matrix of each unit is $m_{1} 、 m_{2} \ldots \ldots m_{n}$,stiffness maxtrix of each unit is $k_{1}$ 、 $k_{2} \ldots \ldots k_{n}$,the kinetic energy of each unit is $T_{1} 、 T_{2} \ldots T_{n}$, and the potential energy of each unit is $U_{1}, U_{2} \ldots U_{n}$.

We define the generalied coordinates in the global coordinates is $z=\left[z_{1}, z_{2}, z_{3}, \ldots \ldots, z_{2 n+1}\right]^{T}$, and get the relations of local coordinate and global coordinate:

$$
y_{i}=A_{i} z, \quad(i=1,2, \ldots \ldots, \mathrm{n})
$$

Where $i$ is unit number, $\mathrm{n}$ is the number of units, ${ }^{A_{i}}$ is relationship matrix,and the transformation matrix:

$$
A=\left[\begin{array}{c}
A_{1} \\
A_{2} \\
\vdots \\
A_{n}
\end{array}\right]
$$

Thus the relationship between the local coordinates and global coordinates can be obtained.

$$
y_{L}=\left\{\begin{array}{c}
y_{1} \\
y_{2} \\
\vdots \\
y_{n}
\end{array}\right\}=A z=A\left[\begin{array}{c}
z_{1} \\
z_{2} \\
\vdots \\
z_{2 n+2}
\end{array}\right]
$$

The potential energy of the system in local coordinates is

$$
\begin{aligned}
& U=U_{1}+U_{2}+\cdots+U_{n} \\
& =\frac{1}{2} \sum_{i=1}^{n} y_{i}^{T} k_{i} y_{i}=\frac{1}{2} y_{L}^{T} k_{L} y_{L}
\end{aligned}
$$

The kinetic energy of the system in local coordinates is

$$
\begin{aligned}
& T=T_{1}+T_{2}+\cdots+T_{n} \\
& =\frac{1}{2} \sum_{i=1}^{n} \dot{y}_{i}^{T} m_{i} \dot{y}_{i}=\frac{1}{2} \dot{y}_{L}^{T} m_{L} \dot{y}_{L}
\end{aligned}
$$

In which,

$$
k_{L}=\left[\begin{array}{cccc}
k_{1} & 0 & 0 & 0 \\
0 & k_{2} & 0 & 0 \\
0 & 0 & k_{3} & 0 \\
0 & 0 & 0 & k_{4}
\end{array}\right]
$$




$$
m_{L}=\left[\begin{array}{cccc}
m_{1} & 0 & 0 & 0 \\
0 & m_{2} & 0 & 0 \\
0 & 0 & m_{3} & 0 \\
0 & 0 & 0 & m_{4}
\end{array}\right]
$$

The relationship of $M$ and $m_{L}$ is

$$
M=A^{T} m_{L} A
$$

The relationship of $K$ and $k_{L}$ is

$$
K=A^{T} k_{L} A
$$

Finally ,the vibration differential equation of transmission is

$$
M \ddot{y}+K y=Q
$$

\section{Case Study}

The paper use a practial example to verify the validity of the model,the parameters of transmission line are shown in chart 1 .

Table 1. The parameters of LGJ - 400/95

\begin{tabular}{cc}
\hline Model & LGJ-400/95 \\
\hline Length & $25.6 \mathrm{~m}$ \\
\hline Cross-sectional area & $501.02 \mathrm{~mm}^{2}$ \\
\hline Mass per unit length & $1860 \mathrm{~kg} / \mathrm{km}$ \\
\hline Outside diameter & $29.14 \mathrm{~mm}$ \\
\hline Coefficient of elasticity & $7.8 \mathrm{e} 10 \mathrm{~N} / \mathrm{mm}^{2}$ \\
\hline Tensile stress & $5.788 \mathrm{e} 7 \mathrm{~N}^{2}$ \\
\hline Bending stiffness of the test & $1059.5 \mathrm{~N} * \mathrm{~m}^{2}$ \\
\hline
\end{tabular}

*NOTE: the bending stiffness data in the table is obtaioned by experiment, the specific method can be find in the References [4].

Modal analysis

The model of first five modal based on the finite element model can be obtained by Matlab.

Table 2. First five natural frequency

\begin{tabular}{cc}
\hline Finite element model & Theoretical value $^{[4]}$ \\
\hline 0.3594299 & 0.3594295 \\
\hline 1.4377419 & 1.4377183 \\
\hline 3.2351340 & 3.2348662 \\
\hline 5.7523666 & 5.7508733 \\
\hline 8.9913810 & 8.9857396 \\
\hline
\end{tabular}



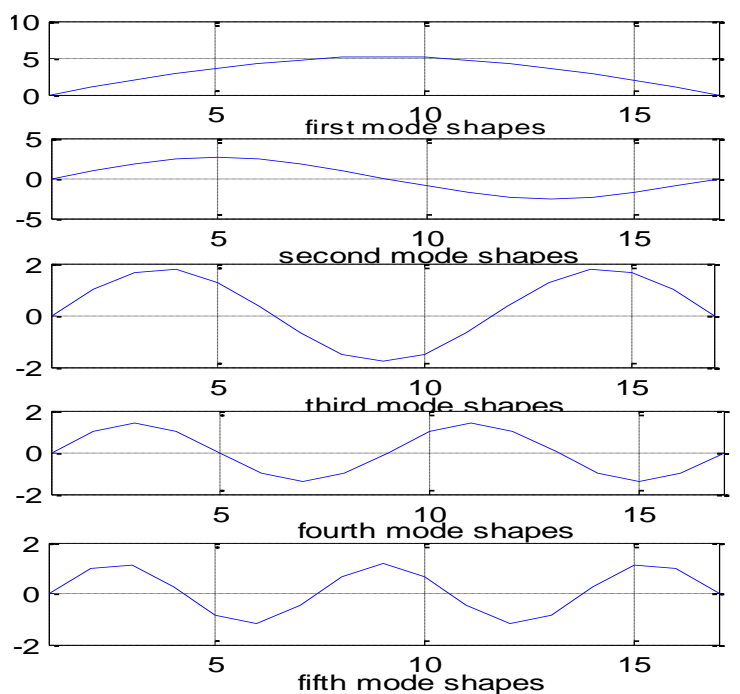

Figure 4. First five mode shapes diagram

Through modal analysis we find the differ of frequencies between theoretical values and the values calculated is less than $1 \%$, and the mode shapes is similar to the theory.

The vibration response analysis

The response of different nodes at different load cases can be obtained by matlab.Now taking the reponse casued by unit and sinusoidal load as example.

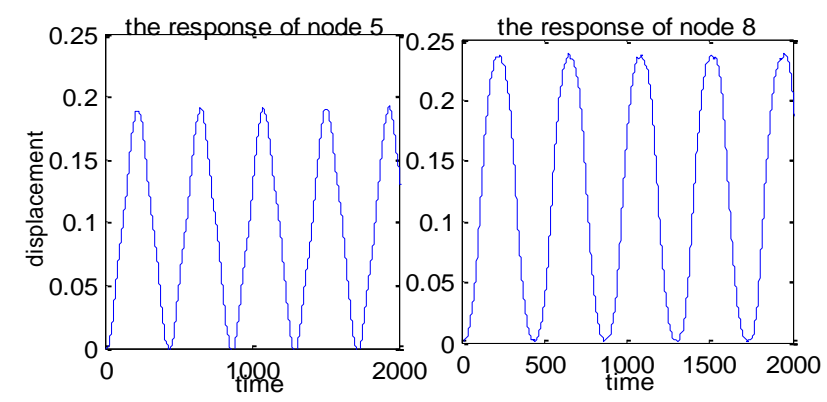

Figure 5 . The response of node 5 and node 8 caused by unit load

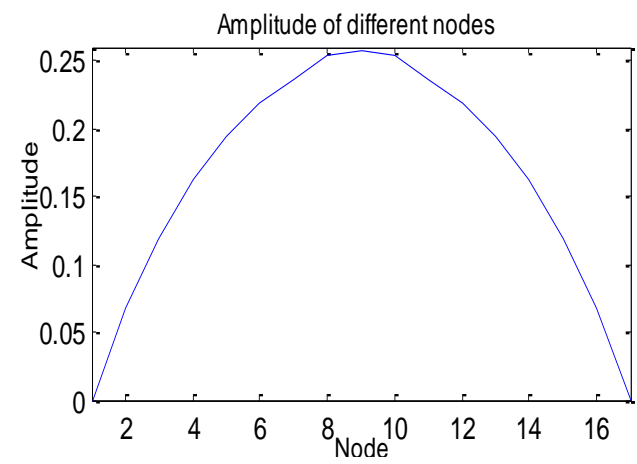

Figure 6. Amplitude of different nodes caused by unit load 

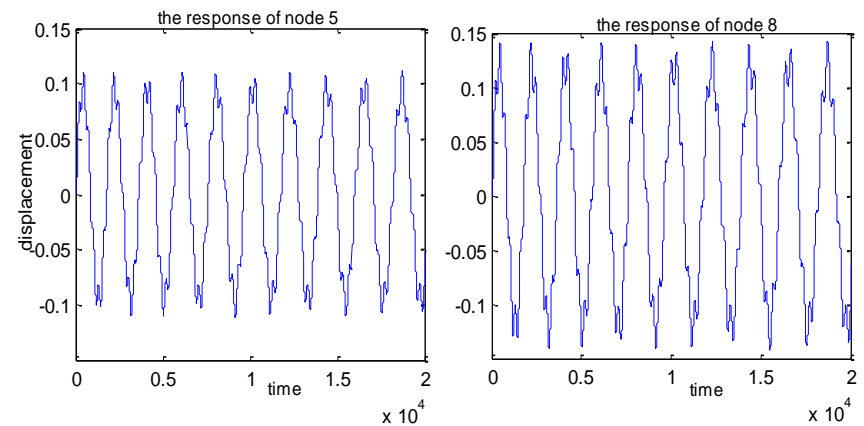

Figure 7. The response of node 5 and node 8 caused by sinusoidal load

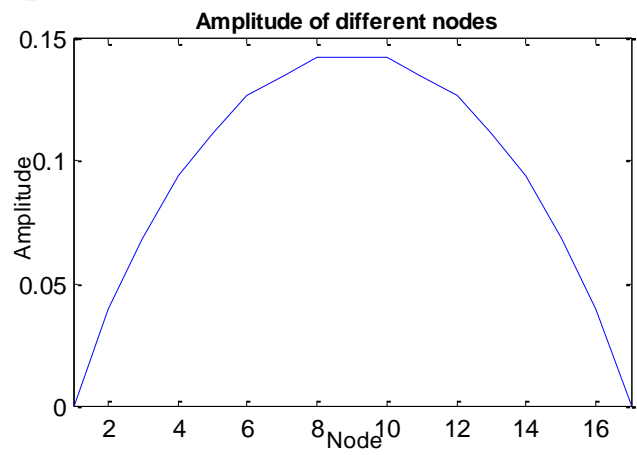

Figure 8. Amplitude of different nodes caused by sinusoidal load

Through analysis above, we find the amplitude along the guide path first increased and than decreases, which is consistent with the actual situation.

\section{Summary}

Considering bending stiffness of transmission and assumed mode method establish the finite element model. then we take analysis based on the model to a specific case study. The results obtained is consistent with the theoretical value. This research can be used to do response analysis and provide reference for the design of overhead transmission lines.

\section{References}

[1] Chen Shuifu, A finite-volume method for large deflection wind-excited response analysis of suspended cables[J].Chinese Journal of Computational Mechanics.2006,23(4):434-439.

[2] ZenPan, Finite Element Essentials[M].Beijing: Higher Education Press, 2009.

[3] Hu Yujin, Wang Zhangqi, Finite Element Analysis and Applications[M].Beijing: Tsinghua University Press, 2009.

[4] Shao Tianxiao, Overhead Transmission Line Wire and Mechanical calculations (second edition)[M].Beijing, China Electric Power Press, 2003.

[5] Guo Yinglong, Transmission Line dancing[M].Beijing, China Electric Power, 2007.

[6] Wang Hong. Study on Aeolian Anti-Vibration of Bundled Conductor of large Crossing Overhead Transmission Lines[D].Baoding: North China Electric Power University,2009.

[7] Zheng Yuqi. Breeze Vibration of Overhead Transmission Lines. Beijing, Water Power Press.1987.7.

[8] Chen Shuifu,Li Changhong.A finite-volume method for large deflection wind-excited response analysis of suspended cable[J].Chinese Journal of Computational Mechanics.2006,23(4):434-439

[9] Claren R,Diana G.Mathematical analysis of transmission line vibration.IEEE Transactions on Power Apparatus and Sytems.1969, PAS_88(12):1741-1771.

[10] He Zeng,Zhao Gaoyu.A finite element for 3D free vibration analysis of bundled power lines with weight hammers[J].Engineering. 